\title{
Analysis for Flow of an Incompressible Brinkman-Type Fluid in Thin Medium with Friction
}

\author{
Soumia Manaa, ${ }^{1}$ Salah Boulaaras $\mathbb{D}^{2,3}$ Hamid Benseridi, ${ }^{1}$ Mourad Dilmi, ${ }^{1}$ \\ and Sultan Alodhaibi ${ }^{2}$ \\ ${ }^{1}$ Applied Mathematics Laboratory, Department of Mathematics, Faculty of Sciences, University of Ferhat ABBAS-Sétif 1, \\ 19000, Algeria \\ ${ }^{2}$ Department of Mathematics, College of Sciences and Arts, ArRass, Qassim University, Saudi Arabia \\ ${ }^{3}$ Laboratory of Fundamental and Applied Mathematics of Oran (LMFAO), University of Oran 1, Oran, 31000 Oran, Algeria
}

Correspondence should be addressed to Salah Boulaaras; s.boularas@qu.edu.sa

Received 23 June 2021; Accepted 2 August 2021; Published 22 September 2021

Academic Editor: Nehad Ali Shah

Copyright ( 2021 Soumia Manaa et al. This is an open access article distributed under the Creative Commons Attribution License, which permits unrestricted use, distribution, and reproduction in any medium, provided the original work is properly cited.

In this paper, we consider the Brinkman equation in the three-dimensional thin domain $\mathbb{Q}^{\varepsilon} \subset \mathbb{R}^{3}$. The purpose of this paper is to evaluate the asymptotic convergence of a fluid flow in a stationary regime. Firstly, we expose the variational formulation of the posed problem. Then, we presented the problem in transpose form and prove different inequalities for the solution $\left(u^{\varepsilon}, p^{\varepsilon}\right)$ independently of the parameter $\varepsilon$. Finally, these estimates allow us to have the limit problem and the Reynolds equation and establish the uniqueness of the solution.

\section{Introduction}

Darcy's law is a physical law that describes the flow of a fluid along a porous medium. It is first named after the French researcher Henri Darcy in 1947 who created a pipeline procedure to supply water around a French town [1]. In 1949, another researcher gave an extension to Darcy's law to which he added the term Brinkman. Thanks to this term, they discovered a new fluid called Darcy-Brinkman [2], which was used to account for transitional flow between boundaries. This model describes a flow in porous media that is fast enough where the drive for flow includes kinetic potential related to fluid velocity, pressure, and gravitational potential. They appear as a mix of Darcy's law and the Stokes equations and extend Darcy's law to account for dissipation of kinetic energy by viscous shear as in the Stokes equation. The average fluid flow through an array of sparse, spherical particles can be modeled via the Brinkman equation $[2,3]$. In addition, Brinkman's equations describe transitions between two flows, one slow and the other fast, where the first type occurs in a porous medium subject to Darcy's law, while the second type occurs in channels subject to Stokes' equa- tions. His equation is

$$
-\mu \nabla^{2} u+\nabla p+\mu\left(\alpha^{\varepsilon}\right)^{2} u=f,
$$

where $\mu$ is the Newtonian fluid viscosity, $\alpha^{\varepsilon}$ is the resistance parameter, which is assumed constant (isotropic), $u$ is the fluid velocity, $p$ is the pressure, and $f$ represents the body force density applied on the fluid.

In recent decades, many authors have studied the Brinkman equation. For instance, Durlofsky and Brady in [4] employ Stokesian dynamics to approximate the fundamental solution or Green's function for flow in random porous media. The authors in [5] investigate a three-dimensional model of flagellar swimming in a Brinkman fluid. In this study, the utility of the Brinkman equation is to model the mean flow rate of the fluid as well as the resistive effects of fibers on the fluid. The fractional Brinkman-type fluid in a channel under the effect of MHD with the Caputo-Fabrizio fractional derivative was studied by Khana et al. in [6]. In the same idea, Liu in [7] has investigated a biobarrier to remove chlorobenzenes from slow-moving ground contaminated water. The goal of the present paper is not only to give 
existence and uniqueness solution of the boundary value problems governed by the Brinkman fluid but also to obtain rigorously the equation describing such a phenomenon in a thin film flow by way of an asymptotic analysis in which a small parameter $\varepsilon$ is the width of the gap. Several authors are interested in the study of asymptotic convergence of Newtonian and non-Newtonian fluids. In the work of Dilmi et al. [8], the authors studied the asymptotic convergence of a Bingham fluid in a thin domain with the Fourier and Tresca boundary condition on the bottom surface. The asymptotic analysis of an incompressible Herschel-Bulkley fluid with friction law is given by [9]. Many works have focused on mechanics of the fluids in a thin domain in the stationary case which is found in the works of $[10,11]$. Other mechanical contact problems similar to incompressible flows in thin medium with friction can be found in $[12,13]$. This paper is divided into four sections: in a first step, we discussed the variational formulation of the problem and demonstrate the results of existence and uniqueness of the weak solution; then, we move on to the study of asymptotic analysis. For this, using the change in the variable $x_{3}$ and unknown news to conduct the study on a domain $\mathbb{Q}$ does not depend on $\varepsilon$. Then, we prove after an explicit work different inequalities for the solution $\left(u^{\varepsilon}, p^{\varepsilon}\right)$ which the thickness becomes infinitely small in the variational formulation. Finally, these estimates allow us to have the limit problem and the Reynolds equation and establish the uniqueness of the solution.

\section{The Problem Statement}

In this section, we give an overview of the thin domain. Next, we introduce the problem considered in this domain. Finally, we explore the theorem of existence and uniqueness of the weak solution.

2.1. The Domain. We denote by $\left(y, y_{3}\right)$ the vector of $\mathbb{R}^{3}$ whose $y=\left(y_{1}, y_{2}\right)$ is the generic vector of $\mathbb{R}^{2}$ and $y_{3} \in \mathbb{R}$.

Let $\Gamma_{b}$ be a domain of the $y$ plane and $h$ be a bounded continuous function defined on $\Gamma_{b}$, with $h$ of class $C^{1}$ such that $0<h_{m} \leq h(y) \leq h_{M}, \forall(y, 0) \in \Gamma_{b}$. The fluid is contained between the lower $\Gamma_{b}$ and the upper surface $\bar{\Gamma}_{u}^{\varepsilon}$ defined by $y_{3}=\varepsilon h(y)$. Let

$$
\mathbb{Q}^{\varepsilon}=\left\{\left(y, y_{3}\right) \in \mathbb{R}^{3} \text { such that } y \in \Gamma_{b} \text {, and } 0<\frac{y_{3}}{\varepsilon}<h(y)\right\},
$$

where $\varepsilon \in] 0,1[$ is a small parameter that will tend to be zero. The boundary of $\mathbb{Q}^{\varepsilon}$ is $\Gamma^{\varepsilon}=\bar{\Gamma}_{b} \cup \bar{\Gamma}_{u}^{\mathcal{\varepsilon}} \cup \bar{\Gamma}_{l}^{\varepsilon}$, where $\bar{\Gamma}_{l}^{\varepsilon}$ is the lateral boundary.

Let $v=\left(v_{1}, v_{2}, v_{3}\right)$ be the unit outward normal to the boundary $\Gamma^{\varepsilon}$. The normal and tangential components of $u^{\varepsilon}$ are given by

$$
u_{v}^{\varepsilon}=u^{\varepsilon} \cdot v=u_{i}^{\varepsilon} v_{i}, u_{\tau_{i}}^{\varepsilon}=u_{i}^{\varepsilon}-u_{v}^{\varepsilon} v_{i}
$$

Similarly, for a regular tensor field $\sigma^{\varepsilon}$, we denote by $\sigma_{v}^{\varepsilon}$ and $\sigma_{\tau}^{\varepsilon}$ the normal and tangential components of $\sigma^{\varepsilon}$ given by

$$
\sigma_{v}^{\varepsilon}=\left(\sigma^{\varepsilon} \cdot v\right) \cdot v=\sigma_{i j}^{\varepsilon} v_{i} v_{j}, \sigma_{\tau_{i}}^{\varepsilon}=\sigma_{i j}^{\varepsilon} v_{j}-\sigma_{v}^{\varepsilon} v_{i}
$$

We introduce the following functional framework:

$$
\begin{gathered}
E^{\varepsilon}=\left\{v \in\left(H^{1}\left(\mathbb{Q}^{\varepsilon}\right)\right)^{3} \text { such that } v=0 \text { on } \Gamma_{u}^{\varepsilon} \cup \Gamma_{l}^{\varepsilon}, v \cdot v=0 \text { on } \Gamma_{b}\right\}, \\
E_{\operatorname{div}}^{\varepsilon}=\left\{v \in E^{\varepsilon} \text { such that } \operatorname{div}(v)=0\right\}, \\
L_{0}^{2}\left(\mathbb{Q}^{\varepsilon}\right)=\left\{q \in L^{2}\left(\mathbb{Q}^{\varepsilon}\right) \text { such that } \int_{\mathbb{Q}^{\varepsilon}} q d y d y_{3}=0\right\} .
\end{gathered}
$$

2.2. The Model Problem. The boundary value problem describing the stationary flow for the incompressible Brinkman fluid is described by the following:

It is supposed that the law of behavior follows the law of Stokes:

$\sigma_{i j}^{\varepsilon}=-p^{\varepsilon} \delta_{i j}+2 \mu d_{i j}\left(u^{\varepsilon}\right), d_{i j}\left(u^{\varepsilon}\right)=\frac{1}{2}\left(\frac{\partial u_{i}^{\varepsilon}}{\partial y_{j}}+\frac{\partial u_{j}^{\varepsilon}}{\partial y_{i}}\right), \quad(1 \leq i, j \leq 3)$,

where $\delta_{i j}$ is the Krönecker symbol.

Our problem comes down to finding the solution $\left(u^{\varepsilon}, p^{\varepsilon}\right)$ which satisfies the following equations:

Problem 1. Find the velocity field $u^{\varepsilon}: \mathbb{Q}^{\varepsilon} \longrightarrow \mathbb{R}^{3}$ and the pressure $p^{\varepsilon}: \mathbb{Q}^{\varepsilon} \longrightarrow \mathbb{R}$, such that

$$
\begin{gathered}
-\mu \Delta u^{\varepsilon}+\nabla p^{\varepsilon}+\mu\left(\alpha^{\varepsilon}\right)^{2} u^{\varepsilon}=f^{\varepsilon} \text { in } \mathbb{Q}^{\varepsilon}, \\
\operatorname{div}\left(u^{\varepsilon}\right)=0 \text { in } \mathbb{Q}^{\varepsilon}, \\
u^{\varepsilon}=0 \text { on } \Gamma_{u}^{\varepsilon}, \\
u^{\varepsilon}=0 \text { on } \Gamma_{l}^{\varepsilon}, \\
u^{\varepsilon} \cdot v=0 \text { on } \Gamma_{b}, \\
\left\{\begin{array}{l}
\left|\sigma_{\tau}^{\varepsilon}\right|<k^{\varepsilon} \Rightarrow u_{\tau}^{\varepsilon}=0, \\
\left|\sigma_{\tau}^{\varepsilon}\right|=k^{\varepsilon} \Rightarrow \exists \beta \geq \operatorname{such} \text { that } u_{\tau}^{\varepsilon}=-\beta \sigma_{\tau}^{\varepsilon},
\end{array}\right.
\end{gathered}
$$

Equation (7) represents the law of conservation of momentum where $f^{\varepsilon}: \mathbb{Q}^{\varepsilon} \longrightarrow \mathbb{R}^{3}$ is the external force. Relation (8) gives the law of behavior of the incompressible fluid. The Dirichlet boundary conditions are given by (9) and (10). The no-flux condition across $\Gamma_{b}$ is given by equation (11). Equation (12) is the condition of the Tresca friction law on the part $\Gamma_{b}$, with $k^{\varepsilon}$ being the friction coefficient (see [13]).

2.3. Weak Variational Formulations. Let $u^{\varepsilon}$ be the solution of (7)-(12), multiplying equation (7) by $\left(\varphi-u^{\varepsilon}\right)$ and then integrating over $\mathbb{Q}^{\varepsilon}$, and using Green's formula and the conditions (9)-(12), we can show that Problem 1 is equivalent to the following variational problem:

Problem 2. Find the pair $\left(u^{\varepsilon}, p^{\varepsilon}\right) \in E_{\text {div }}^{\varepsilon} \times L_{0}^{2}\left(\mathbb{Q}^{\varepsilon}\right)$, such that $a\left(u^{\varepsilon}, \varphi-u^{\varepsilon}\right)-\left(p^{\varepsilon}, \operatorname{div} \varphi\right)+j(\varphi)-j\left(u^{\varepsilon}\right) \geq\left(f^{\varepsilon}, \varphi-u^{\varepsilon}\right), \quad \forall \varphi \in E^{\varepsilon}$, 
where

$$
\begin{gathered}
a\left(u^{\varepsilon}, \varphi-u^{\varepsilon}\right)=\int_{\mathbb{Q}^{\varepsilon}} 2 \mu d_{i j}\left(u^{\varepsilon}\right) d_{i j}\left(\varphi_{i}-u_{i}^{\varepsilon}\right) d y d y_{3}+\mu \int_{\mathbb{Q}^{\varepsilon}}\left(\alpha^{\varepsilon}\right)^{2} u_{i}^{\varepsilon}\left(\varphi_{i}-u_{i}^{\varepsilon}\right) d y d y_{3}, \\
\left(p^{\varepsilon}, \operatorname{div} \varphi\right)=\int_{\mathbb{Q}^{\varepsilon}} p^{\varepsilon} \delta_{i j} \frac{\partial}{\partial y_{j}}\left(\varphi_{i}-u_{i}^{\varepsilon}\right) d y d y_{3}, \\
j(\varphi)=\int_{\Gamma_{b}} k^{\varepsilon}|\varphi| d y,\left(f^{\varepsilon}, \varphi-u^{\varepsilon}\right)=\int_{\mathbb{Q}^{\varepsilon}} f_{i}^{\varepsilon}\left(\varphi_{i}-u_{i}^{\varepsilon}\right) d y d y_{3} .
\end{gathered}
$$

Theorem 3. If $k^{\varepsilon} \in L_{+}^{\infty}\left(\Gamma_{b}\right)$ and $f^{\varepsilon} \in\left(L^{2}\left(\mathbb{Q}^{\varepsilon}\right)\right)^{3}$, then there exists a unique $u^{\varepsilon} \in E_{\text {div }}^{\varepsilon}$ and $p^{\varepsilon} \in L_{0}^{2}\left(\mathbb{Q}^{\varepsilon}\right)$ (to an additive constant) solution to problem (13).

Proof. Since our goal in this work is to prove the asymptotic convergence of the problem posed, we only give the steps followed for the proof of this theorem. Let $\varphi \in E_{\text {div }}^{\varepsilon}$ in (13); we have the following:

Find $u^{\varepsilon} \in E_{\text {div }}^{\varepsilon}$, such that

$$
a\left(u^{\varepsilon}, \varphi-u^{\varepsilon}\right)+j(\varphi)-j\left(u^{\varepsilon}\right) \geq\left(f^{\varepsilon}, \varphi-u^{\varepsilon}\right), \quad \forall \varphi \in E_{\text {div }}^{\varepsilon} .
$$

Using the Cauchy-Schwartz inequality and $\sum_{i, j=1}^{3}$ $\left|d_{i j}\left(u^{\varepsilon}\right)\right|^{2} \leq\left|\nabla u^{\varepsilon}\right|^{2}$, we obtain that the bilinear from $a(.,$.$) is$ continuous:

$$
\begin{aligned}
\left|a\left(u^{\varepsilon}, v\right)\right| & =\int_{\mathbb{Q}^{\varepsilon}} 2 \mu d_{i j}\left(u^{\varepsilon}\right) d_{i j}(v) d y d y_{3}+\int_{\mathbb{Q}^{\varepsilon}} \mu\left(\alpha^{\varepsilon}\right)^{2} u^{\varepsilon} v d y d y_{3} \\
& \leq\left(2 \mu+\mu\left(\alpha^{\varepsilon}\right)^{2}\right)\left\|u^{\varepsilon}\right\|_{H^{1}(\mathbb{Q})}\|v\|_{H^{1}(\mathbb{Q})}, \quad \forall\left(u^{\varepsilon}, v\right) \in\left(E_{\text {div }}^{\varepsilon}\right)^{2} .
\end{aligned}
$$

By Korn's inequality, we obtain

$$
\begin{aligned}
a\left(u^{\varepsilon}, u^{\varepsilon}\right) & =2 \mu \sum_{i, j=1}^{3}\left\|d_{i j}\left(u^{\varepsilon}\right)\right\|_{L^{2}\left(\mathbb{Q}^{\varepsilon}\right)}^{2}+\mu\left(\alpha^{\varepsilon}\right)^{2}\left\|u^{\varepsilon}\right\|_{L^{2}\left(\mathbb{Q}^{\varepsilon}\right)}^{2} \\
& \geq \min \left(2 \mu C_{k}, \mu\left(\alpha^{\varepsilon}\right)^{2}\right)\left\|u^{\varepsilon}\right\|_{H^{1}(\mathbb{Q})}^{2}, \quad \forall u^{\varepsilon} \in E_{\text {div }}^{\varepsilon},
\end{aligned}
$$

where $C_{k}>0$ independent of $\varepsilon$. We deduce that $a(.,$.$) is coer-$ cive on $E_{\text {div }}^{\varepsilon} \times E_{\text {div }}^{\varepsilon}$. Moreover, $j$ is convex and continuous on $E_{\text {div }}^{\varepsilon}$. This ensures the existence and uniqueness of $u^{\varepsilon} \in E_{\text {div }}^{\varepsilon}$ satisfying the variational inequality (15). In addition, using the techniques of [14], we can prove the existence of $p^{\varepsilon} \in L_{0}^{2}$ $\left(\mathbb{Q}^{\varepsilon}\right)$ for which $\left(u^{\varepsilon}, p^{\varepsilon}\right)$ is a solution of (13).

\section{Dilatation in the Variable $y_{3}$}

In this section, we use the dilatation in the variable $y_{3}$ given by $y_{3}=z \varepsilon$; then, our problem will be defined on a domain $\mathbb{Q}$ which does not depend on $\varepsilon$ given by

$$
\mathbb{Q}=\left\{(y, z) \in \mathbb{R}^{3} \text { such that } y \in \Gamma_{b} \text { and } 0<z<h(y)\right\},
$$

and its boundary $\Gamma=\bar{\Gamma}_{1} \cup \bar{\Gamma}_{L} \cup \bar{\Gamma}_{b}$.
After this change, here are the new functions defined on the fixed domain $\mathbb{Q}$ :

$$
\left\{\begin{array}{l}
\widehat{u}_{i}^{\varepsilon}(y, z)=u_{i}^{\varepsilon}\left(y, y_{3}\right), \quad(i=1,2), \\
\widehat{u}_{3}^{\varepsilon}(y, z)=\varepsilon^{-1} u_{3}^{\varepsilon}\left(y, y_{3}\right), \\
p \wedge^{\varepsilon}(y, z)=\varepsilon^{2} p^{\varepsilon}\left(y, y_{3}\right) .
\end{array}\right.
$$

Likewise for new data,

$$
\left\{\begin{array}{l}
\widehat{f}(y, z)=\varepsilon^{2} f^{\varepsilon}\left(y, y_{3}\right), \\
\widehat{\alpha}=\varepsilon \alpha^{\varepsilon} \\
\widehat{k}=\varepsilon k^{\varepsilon}
\end{array}\right.
$$

We denote by

$$
\begin{gathered}
E=\left\{\hat{v} \in\left(H^{1}(\mathbb{Q})\right)^{3}: \widehat{v}=0 \text { on } \Gamma_{u} \cup \Gamma_{l}, \widehat{v} \cdot v=0 \text { on } \Gamma_{b}\right\}, \\
E_{\operatorname{div}}=\{\hat{v} \in E: \operatorname{div}(\widehat{v})=0\}, \\
L_{0}^{2}(\mathbb{Q})=\left\{q \in L^{2}(\mathbb{Q}): \int_{\mathbb{Q}} q d y d y_{3}=0\right\}, \\
V_{z}=\left\{\Phi=\left(\Phi_{1}, \Phi_{2}\right) \in\left(L^{2}(\mathbb{Q})\right)^{2}: \frac{\partial \Phi_{i}}{\partial z} \in L^{2}(\mathbb{Q}), \quad \Phi=0 \text { on } \Gamma_{u} \cup \Gamma_{l}\right\},
\end{gathered}
$$

the Banach space with the norm

$$
\|\Phi\|_{V_{z}}=\left(\sum_{i=1}^{2}\left(\left\|\Phi_{i}\right\|_{L^{2}(\mathbb{Q})}^{2}+\left\|\frac{\partial \Phi_{i}}{\partial z}\right\|_{L^{2}(\mathbb{Q})}^{2}\right)\right)^{1 / 2} .
$$

According to (19) and (20), then Problem 2 leads to the following:

Problem 4. Find $\left(u \wedge^{\varepsilon}, p \wedge^{\varepsilon}\right) \in E \times L_{0}^{2}(\mathbb{Q})$, such that

$$
\begin{aligned}
& \int_{\mathbb{Q}} q \operatorname{div}\left(u \wedge^{\varepsilon}\right) d y d z=0, \forall q \in L_{0}^{2}(\mathbb{Q}) \\
& \sum_{i, j=1}^{2} \int_{\mathbb{Q}}\left(\varepsilon^{2} \mu\left(\frac{\partial \hat{u}_{i}^{\varepsilon}}{\partial y_{j}}+\frac{\partial \widehat{u}_{j}^{\varepsilon}}{\partial y_{i}}\right)-p \wedge^{\varepsilon} \delta_{i j}\right) \frac{\partial}{\partial y_{j}}\left(\widehat{\varphi}_{i}-\widehat{u}_{i}^{\varepsilon}\right) d y d z \\
& +\sum_{i=1}^{2} \int_{\mathbb{Q}} \mu\left(\frac{\partial \widehat{u}_{i}^{\varepsilon}}{\partial z}+\varepsilon^{2} \frac{\partial \widehat{u}_{3}^{\varepsilon}}{\partial y_{i}}\right) \frac{\partial}{\partial z}\left(\widehat{\varphi}_{i}-\widehat{u}_{i}^{\varepsilon}\right) d y d z \\
& +\sum_{j=1}^{2} \int_{\mathbb{Q}} \mu\left(\varepsilon^{2} \frac{\partial \widehat{u}_{3}^{\varepsilon}}{\partial y_{j}}+\frac{\partial \hat{u}_{i}^{\varepsilon}}{\partial z}\right) \varepsilon^{2} \frac{\partial}{\partial y_{i}}\left(\widehat{\varphi}_{3}-\widehat{u}_{3}^{\varepsilon}\right) d y d z \\
& +\int_{\mathbb{Q}}\left(2 \mu \varepsilon^{2} \frac{\partial \widehat{u}_{3}^{\varepsilon}}{\partial z}-p \wedge^{\varepsilon}\right) \frac{\partial}{\partial z}\left(\widehat{\varphi}_{3}-\widehat{u}_{3}^{\varepsilon}\right) d y d z \\
& +\sum_{i=1}^{2} \mu \alpha \wedge^{2} \int_{\mathbb{Q}} \widehat{u}_{i}^{\varepsilon}\left(\widehat{\varphi}_{i}-\widehat{u}_{i}^{\varepsilon}\right) d y d z+\varepsilon^{2} \mu \alpha \wedge^{2} \int_{\mathbb{Q}} \widehat{u}_{3}^{\varepsilon}\left(\widehat{\varphi}_{3}-\widehat{u}_{3}^{\varepsilon}\right) d y d z \\
& +\int_{\Gamma_{b}} \widehat{k}\left(|\widehat{\varphi}|-\left|u \wedge^{\varepsilon}\right|\right) d y \geq \sum_{i=1}^{2} \int_{\mathbb{Q}} \widehat{f}_{i}\left(\widehat{\varphi}_{i}-\widehat{u}_{i}^{\varepsilon}\right) d y d z \\
& +\varepsilon \int_{\mathbb{Q}} \widehat{f}_{3}\left(\widehat{\varphi}_{3}-\widehat{u}_{3}^{\varepsilon}\right) d y d z, \forall \varphi \in E .
\end{aligned}
$$


In the next, we will do the estimates of velocity $u \wedge^{\varepsilon}$ and then on the pressure $p \wedge^{\varepsilon}$ solution of our variational problem in the fixed domain.

Theorem 5. Assuming (19) and (20), the following estimate on $u \wedge^{\varepsilon}$ is satisfied:

$$
\begin{aligned}
& \sum_{i, j=1}^{2} \varepsilon^{2}\left\|\frac{\partial \widehat{u}_{i}^{\varepsilon}}{\partial y_{j}}\right\|_{L^{2}(\mathbb{Q})}^{2}+\sum_{i=1}^{2}\left\|\frac{\partial \widehat{u}_{i}^{\varepsilon}}{\partial z}\right\|_{L^{2}(\mathbb{Q})}^{2} \\
& \quad+\varepsilon^{2}\left[\left\|\frac{\partial \widehat{u}_{3}^{\varepsilon}}{\partial z}\right\|_{L^{2}(\mathbb{Q})}^{2}+\varepsilon^{2} \sum_{i=1}^{2}\left\|\frac{\partial \hat{u}_{3}^{\varepsilon}}{\partial y_{i}}\right\|_{L^{2}(\mathbb{Q})}^{2}\right]+\sum_{i=1}^{2}\left\|\hat{u}_{i}^{\varepsilon}\right\|_{L^{2}(\mathbb{Q})}^{2}+\varepsilon^{2}\left\|\hat{u}_{3}^{\varepsilon}\right\|_{L^{2}(\mathbb{Q})}^{2} \leq C .
\end{aligned}
$$

Proof. Let $u^{\varepsilon}$ be the solution of (15), we choose $\varphi=0$, and we obtain

$$
a\left(u^{\varepsilon}, u^{\varepsilon}\right) \leq \int_{\mathbb{Q}} f^{\varepsilon} u^{\varepsilon} d y d y_{3}
$$

Now, $a\left(u^{\varepsilon}, u^{\varepsilon}\right)=2 \mu \int_{\mathbb{Q}^{\varepsilon}} d_{i j}\left(u^{\varepsilon}\right) d_{i j}\left(u^{\varepsilon}\right) d y d y_{3}+\mu \int_{\mathbb{Q}^{\varepsilon}} u_{i}^{\varepsilon} u_{i}^{\varepsilon} d y$ $d y_{3}$. By Korn's inequality, there exists a constant $C_{k}>0$ independent of $\varepsilon$, such that

$$
2 \mu \int_{\mathbb{Q}^{\varepsilon}} d_{i j}\left(u^{\varepsilon}\right) d_{i j}\left(u^{\varepsilon}\right) d y d y_{3} \geq 2 \mu C_{k}\left\|\nabla u^{\varepsilon}\right\|_{L^{2}\left(\mathbb{Q}^{\varepsilon}\right)}^{2}
$$

We apply the Cauchy-Schwarz inequality and then the Young inequality; we obtain the following:

$$
\left(f^{\varepsilon}, u^{\varepsilon}\right) \leq \frac{h_{M}^{2} \varepsilon^{2}}{4 \mu C_{k}}\left\|f^{\varepsilon}\right\|_{L^{2}\left(\mathbb{Q}^{\varepsilon}\right)}^{2}+\frac{C_{k} \mu}{h_{M}^{2} \varepsilon^{2}}\left\|u^{\varepsilon}\right\|_{L^{2}\left(\mathbb{Q}^{\varepsilon}\right)}^{2} .
$$

By the Poincaré inequality, we give

$$
\mu C_{k}\left\|\nabla u^{\varepsilon}\right\|_{L^{2}\left(\mathbb{Q}^{\varepsilon}\right)}^{2}+\mu\left(\alpha^{\varepsilon}\right)^{2}\left\|u^{\varepsilon}\right\|_{L^{2}\left(\mathbb{Q}^{\varepsilon}\right)} \leq \frac{h_{M}^{2} \varepsilon^{2}}{4 \mu C_{k}}\left\|f^{\varepsilon}\right\|_{L^{2}\left(\mathbb{Q}^{\varepsilon}\right)}^{2} .
$$

As $\varepsilon^{2}\left\|f^{\varepsilon}\right\|_{L^{2}\left(\mathbb{Q}^{2}\right)}^{2}=\varepsilon^{-1}\|\widehat{f}\|_{L^{2}(\mathbb{Q})}^{2}, \varepsilon \leq 1$, then we multiply the last equation by $\varepsilon$; we obtain

$$
\min \left(\mu C_{k}, \mu \alpha \wedge^{2}\right)\left(\varepsilon\left\|\nabla u^{\varepsilon}\right\|_{L^{2}\left(\mathbb{Q}^{\varepsilon}\right)}^{2}+\left\|u^{\varepsilon}\right\|_{L^{2}\left(\mathbb{Q}^{\varepsilon}\right)}^{2}\right) \leq \frac{h_{M}^{2}}{4 \mu C_{k}}\|\hat{f}\|_{L^{2}(\mathbb{Q})}^{2}
$$

Writing this equation in terms of $u \wedge^{\varepsilon}$, we deduce (25).

Theorem 6. Assuming (19), the following estimates on $p^{\varepsilon}$ are satisfied:

$$
\left\|\frac{\partial p \wedge^{\varepsilon}}{\partial z}\right\|_{H^{-1}(\mathbb{Q})} \leq \varepsilon C_{1}
$$

$$
\left\|\frac{\partial \wedge^{\varepsilon}}{\partial y_{i}}\right\|_{H^{-1}(\mathbb{Q})} \leq C_{2}, \quad(i=1,2)
$$

where $C_{1}$ and $C_{2}$ denote the constants independent of $\varepsilon$.

Proof. Let $\psi \in H_{0}^{1}(\mathbb{Q})$; putting in (24) $\widehat{\varphi}_{i}=\widehat{u}_{i}^{\varepsilon}($ for $i=1,2)$ and $\widehat{\varphi}_{3}=\widehat{u}_{3}^{\varepsilon} \pm \psi$, we deduce

$$
\begin{aligned}
& \sum_{i=1}^{2} \int_{\mathbb{Q}} \varepsilon^{2} \mu\left(\varepsilon^{2} \frac{\partial \widehat{u}_{3}^{\varepsilon}}{\partial y_{j}}+\frac{\partial \hat{u}_{i}^{\varepsilon}}{\partial z}\right) \frac{\partial \psi}{\partial y_{i}} d y d z+\int_{\mathbb{Q}}\left(2 \varepsilon^{2} \mu \frac{\partial \widehat{u}_{3}^{\varepsilon}}{\partial z}-p \wedge^{\varepsilon}\right) \frac{\partial \psi}{\partial z} d y d z \\
& \quad+\varepsilon^{2} \mu \alpha \wedge^{2} \int_{\mathbb{Q}} \widehat{u}_{3}^{\varepsilon} \psi d y d z=\varepsilon \int_{\mathbb{Q}} \widehat{f}_{3} \psi d y d z .
\end{aligned}
$$

Therefore,

$$
\begin{aligned}
\int_{\mathbb{Q}} p \wedge^{\varepsilon} \frac{\partial \psi}{\partial z} d y d z= & \sum_{i=1}^{2} \int_{\mathbb{Q}} \varepsilon^{2} \mu\left(\varepsilon^{2} \frac{\partial \widehat{u}_{3}^{\varepsilon}}{\partial y_{j}}+\frac{\partial \widehat{u}_{i}^{\varepsilon}}{\partial z}\right) \frac{\partial \psi}{\partial y_{i}} d y d z \\
& +\int_{\mathbb{Q}} 2 \varepsilon^{2} \mu \frac{\partial \widehat{u}_{3}^{\varepsilon}}{\partial z} \frac{\partial \psi}{\partial z} d y d z+\varepsilon^{2} \mu \alpha \wedge^{2} \int_{\mathbb{Q}} \widehat{u}_{3}^{\varepsilon} \psi d y d z \\
& -\varepsilon \int_{\mathbb{Q}} \widehat{f}_{3} \psi d y d z .
\end{aligned}
$$

Using Green's formula and the Cauchy-Schwarz inequality, we have

$$
\begin{aligned}
\left|\int_{Q} \frac{\partial p \wedge^{\varepsilon}}{\partial z} \psi d x d z\right| \leq & {\left[\varepsilon^{4} \mu\left(\sum_{i=1}^{2}\left\|\frac{\partial u \wedge_{3}^{\varepsilon}}{\partial x_{j}}\right\|_{L^{2}(\mathbb{Q})}^{2}\right)^{1 / 2}+\varepsilon^{2} \mu\left(\sum_{i=1}^{2}\left\|\frac{\partial \wedge_{j}^{\varepsilon}}{\partial z}\right\|_{L^{2}(\mathbb{Q})}^{2}\right)^{1 / 2}\right.} \\
& \left.+2 \varepsilon^{2} \mu\left\|\frac{\partial \hat{u}_{3}^{\varepsilon}}{\partial z}\right\|_{L^{2}(\mathbb{Q})}+\varepsilon^{2} \mu \alpha \wedge^{2}\left\|\hat{u}_{3}^{\varepsilon}\right\|_{L^{2}(\mathbb{Q})}+\varepsilon\left\|\hat{f}_{3}\right\|_{L^{2}(\mathbb{Q})}\right]\|\psi\|_{H_{0}^{\prime}(\mathbb{Q})} .
\end{aligned}
$$

From Theorem 5, we deduce the inequality (31).

Taking in (24) $\widehat{\varphi}_{1}^{\varepsilon}=\widehat{u}_{1}^{\varepsilon} \pm \psi, \psi$ in $H_{0}^{1}(\mathbb{Q}) ; \widehat{\varphi}_{i}=\widehat{u}_{i}^{\varepsilon}, i=2,3$; we have

$$
\begin{aligned}
\int_{\mathbb{Q}} p \wedge^{\varepsilon} \frac{\partial \psi}{\partial y_{1}} d y d z= & \int_{\mathbb{Q}} \mu\left(\frac{\partial \widehat{u}_{1}^{\varepsilon}}{\partial z}+\varepsilon^{2} \frac{\partial \widehat{u}_{3}^{\varepsilon}}{\partial y_{1}}\right) \frac{\partial \psi}{\partial z} d y d z \\
& +\int_{\mathbb{Q}} 2 \varepsilon^{2} \mu \frac{\partial \widehat{u}_{1}^{\varepsilon}}{\partial y_{1}} \frac{\partial \psi}{\partial y_{1}} d y d z \\
& +\int_{\mathbb{Q}} \varepsilon^{2} \mu\left(\frac{\partial \widehat{u}_{1}^{\varepsilon}}{\partial y_{2}}+\frac{\partial \widehat{u}_{2}^{\varepsilon}}{\partial y_{1}}\right) \frac{\partial \psi}{\partial y_{2}} d y d z \\
& +\mu \alpha \wedge^{2} \int_{\mathbb{Q}} \widehat{u}_{1}^{\varepsilon} \psi d y d z-\int_{\mathbb{Q}} \widehat{f}_{1} \psi d y d z .
\end{aligned}
$$

In the same way, by the chosen $\widehat{\varphi}_{1}=\widehat{u}_{1}^{\varepsilon}, \widehat{\varphi}_{3}=\widehat{u}_{3}^{\varepsilon}$, and 
$\widehat{\varphi}_{2}=\widehat{u}_{2}^{\varepsilon} \pm \psi, \psi$ in $H_{0}^{1}(\mathbb{Q})$, we have

$$
\begin{aligned}
\int_{\mathbb{Q}} p \wedge^{\varepsilon} \frac{\partial \psi}{\partial y_{2}} d y d z= & \int_{\mathbb{Q}} \mu\left(\frac{\partial \widehat{u}_{2}^{\varepsilon}}{\partial z}+\varepsilon^{2} \frac{\partial \widehat{u}_{3}^{\varepsilon}}{\partial y_{2}}\right) \frac{\partial \psi}{\partial z} d y d z \\
& +\int_{\mathbb{Q}} 2 \varepsilon^{2} \mu \frac{\partial \widehat{u}_{2}^{\varepsilon}}{\partial y_{2}} \frac{\partial \psi}{\partial y_{2}} d y d z \\
& +\int_{\mathbb{Q}} \varepsilon^{2} \mu\left(\frac{\partial \widehat{u}_{1}^{\varepsilon}}{\partial y_{2}}+\frac{\partial \widehat{u}_{2}^{\varepsilon}}{\partial y_{1}}\right) \frac{\partial \psi}{\partial y_{1}} d y d z \\
& +\mu \alpha \wedge^{2} \int_{\mathbb{Q}} \widehat{u}_{2}^{\varepsilon} \psi d y d z-\int_{\mathbb{Q}} \widehat{f}_{2} \psi d y d z
\end{aligned}
$$

We use the same technical in (36) and (37) to obtain inequality (32).

Thanks to the estimations (25)-(32), we have the following convergence result:

Theorem 7. Suppose that the estimations (25)-(32) hold. There exists $\left(u^{*}, p^{*}\right)=\left(\left(u_{1}^{*}, u_{2}^{*}\right), p^{*}\right)$ in $V_{z} \times L_{0}^{2}(\mathbb{Q})$ such that we have the following:

$$
\begin{gathered}
\widehat{u}_{i}^{\varepsilon} \rightarrow u_{i}^{*} \quad \text { in } V_{z}, \quad 1 \leq i \leq 2, \\
\varepsilon \frac{\partial \widehat{u}_{i}^{\varepsilon}}{\partial y_{j}} \rightarrow 0 \quad \text { in } L^{2}(\mathbb{Q}), \quad 1 \leq i, j \leq 2, \\
\varepsilon \frac{\partial \widehat{u}_{3}^{\varepsilon}}{\partial z} \rightarrow 0 \quad \text { in } L^{2}(\mathbb{Q}), \\
\varepsilon^{2} \frac{\partial \widehat{u}_{3}^{\varepsilon}}{\partial y_{i}} \rightarrow 0 \quad \text { in } L^{2}(\mathbb{Q}), \quad 1 \leq i \leq 2, \\
\varepsilon \widehat{u}_{3}^{\varepsilon} \rightarrow 0 \quad \text { in } L^{2}(\mathbb{Q}), \\
p \wedge^{\varepsilon} \rightarrow p^{*} \quad \text { in } L_{0}^{2}(\mathbb{Q}) .
\end{gathered}
$$

Proof. Using the estimate (25) and Poincaré's inequality in the domain $\mathbb{Q}$ :

$$
\left\|\frac{\partial \widehat{u}_{i}^{\varepsilon}}{\partial z}\right\|_{L^{2}(\mathbb{Q})}^{2}+\left\|\widehat{u}_{i}^{\varepsilon}\right\|_{L^{2}(\mathbb{Q})}^{2} \leq C(i=1,2)
$$

we deduce (38). Also, equations (39)-(42) follow from (25) and $\operatorname{div}\left(u \wedge^{\varepsilon}\right)=0$. Finally, the weakly convergences (43) follow from (31) and (32) and [15].

\section{Study of the Limit Problem}

To reach the desired goal, we need in the rest of this paragraph the results of previous convergences. find

We go to the limit in (24), and using (23) $(\varepsilon \longrightarrow 0)$, we

$$
\begin{aligned}
& \sum_{i=1}^{2} \int_{\mathbb{Q}} \mu \frac{\partial u_{i}^{*}}{\partial z} \frac{\partial}{\partial z}\left(\widehat{\varphi}_{i}-u_{i}^{*}\right) d y d z-\int_{\mathbb{Q}} p^{*}\left(\frac{\partial \widehat{\varphi}_{1}}{\partial y_{1}}+\frac{\partial \widehat{\varphi}_{2}}{\partial y_{2}}\right) d y d z \\
& \quad+\sum_{i=1}^{2} \mu \widehat{\alpha} \int_{\mathbb{Q}} u_{i}^{*}\left(\widehat{\varphi}_{i}-u_{i}^{*}\right) d y d z+\widehat{k} \int_{\Gamma_{b}}\left(\widehat{\varphi}-u^{*}\right) d y \geq\left(\widehat{f},\left(\widehat{\varphi}-u^{*}\right)\right) .
\end{aligned}
$$

Moreover, if

$$
\int_{\mathbb{Q}}\left(\widehat{\varphi}_{1} \frac{\partial \theta}{\partial y_{1}}(y)+\widehat{\varphi}_{2} \frac{\partial \theta}{\partial y_{2}}(y)\right) d y d z=0 \forall \theta \in C_{0}^{1}\left(\Gamma_{b}\right)
$$

then

$$
\begin{aligned}
& \sum_{i=1}^{2} \int_{\mathbb{Q}} \mu \frac{\partial u_{i}^{*}}{\partial z} \frac{\partial}{\partial z}\left(\widehat{\varphi}_{i}-u_{i}^{*}\right) d y d z \\
& \quad+\sum_{i=1}^{2} \mu \alpha \wedge^{2} \int_{\mathbb{Q}} u_{i}^{*}\left(\widehat{\varphi}_{i}-u_{i}^{*}\right) d y d z+\widehat{k} \int_{\Gamma_{b}}\left(|\widehat{\varphi}|-\left|u^{*}\right|\right) d y \\
& \quad \geq \sum_{i=1}^{2} \int_{\mathbb{Q}} \widehat{f}_{i}\left(\widehat{\varphi}_{i}-u_{i}^{*}\right) d y d z, \forall \widehat{\varphi} \in \Pi(E),
\end{aligned}
$$

where

$\Pi(E)=\left\{\psi=\left(\widehat{\psi}_{1}, \widehat{\psi}_{2}\right) \in\left(H^{1}(\mathbb{Q})\right)^{2}: \exists \widehat{\psi}_{3}\right.$ such that $\left.\psi=\left(\widehat{\psi}_{1}, \widehat{\psi}_{2}, \widehat{\psi}_{3}\right) \in E\right\}$.

Lemma 8. If the estimations (25)-(32) hold, then $\left(u^{*}, p^{*}\right)$ satisfy

$$
\mu \int_{\mathbb{Q}}\left|\frac{\partial u^{*}}{\partial z}\right|^{2} d y d z+\mu \alpha \wedge^{2} \int_{\mathbb{Q}}\left|u^{*}\right|^{2} d y d z+\widehat{k} \int_{\Gamma_{b}}\left|u^{*}\right| d y-\int_{\mathbb{Q}} \widehat{f} u^{*} d y d z=0
$$

$$
\begin{aligned}
& \mu \int_{\mathbb{Q}} \frac{\partial u^{*}}{\partial z} \frac{\partial \widehat{\varphi}}{\partial z} d y d z+\mu \alpha \wedge^{2} \int_{\mathbb{Q}} u^{*} \widehat{\varphi} d y d z+\widehat{k} \int_{\Gamma_{b}}|\widehat{\varphi}| d y \\
& \quad \geq \int_{\mathbb{Q}} \widehat{f} \widehat{\varphi} d y d z, \forall \widehat{\varphi} \in \Sigma(E),
\end{aligned}
$$

where $\Sigma(E)=\{\bar{\varphi} \in \Pi(E): \bar{\varphi}$ satisfies condition $(32)\}$.

Proof. As in [16], by choosing $\widehat{\varphi}=0$ in (47), we find that the second member of this inequality is an upper bound of its first member. Then, for $\widehat{\varphi}=2 u^{*}$ in the same inequality, we get the reverse, which therefore gives equality (49). Now, for $\widehat{\varphi}=u^{*}+\widehat{\varphi}$ in (47), we deduce directly the inequality (50). It is shown reciprocally that (49) and (50) imply (47). 
For this, we consider the mapping:

$$
\begin{gathered}
\Lambda: \Sigma(E) \longrightarrow L^{1}\left(\Gamma_{b}\right)^{2}, \\
\tilde{\varphi} \longrightarrow \Lambda(\widehat{\varphi})=\tilde{k} \tilde{\varphi} .
\end{gathered}
$$

Let us define $F$ as

$$
F(\widehat{k} \widehat{\varphi})=\mu \int_{\mathbb{Q}} \frac{\partial u^{*}}{\partial z} \frac{\partial \widehat{\varphi}}{\partial z} d y d z+\mu \alpha \wedge^{2} \int_{\mathbb{Q}} u^{*} \widehat{\varphi} d y d z-\int_{\mathbb{Q}} \widehat{f} \widehat{\varphi} d y d z
$$

For all $\widehat{\psi} \in \Sigma(E)$, we choose $\widehat{\varphi}=-\widehat{\psi}$ in (49):

$$
|F(\widehat{k} \widehat{\psi})| \leq \int_{\Gamma_{b}} \widehat{k}|\widehat{\psi}| d y .
$$

By (53), $F$ is a continuous linear function on the subspace of $\left(L^{1}\left(\Gamma_{b}\right)\right)^{2}$ which is the image of $\Sigma(V)$ by $\Lambda$. Then, by the Han-Banach theorem, there exists $\chi \in\left(L^{\infty}\left(\Gamma_{b}\right)\right)^{2}$, with $|\chi|$ $\leq 1$, such that

$$
F(\widehat{k} \widehat{\psi})=-\int_{\Gamma_{b}} \chi \widehat{k} \widehat{\psi} d y
$$

In particular, from (50) and (54), we get

$$
\int_{\Gamma_{b}} \widehat{k}\left|u^{*}\right| d y=\int_{\Gamma_{b}} \chi \widehat{k} u^{*} d y
$$

Also,

$\mu \int_{\mathbb{Q}} \frac{\partial u^{*}}{\partial z} \frac{\partial \widehat{\psi}}{\partial z} d y d z+\mu \alpha \wedge^{2} \int_{\mathbb{Q}} u^{*} \widehat{\psi} d y d z-\int_{\mathbb{Q}} \widehat{f} \widehat{\psi} d y d z+\int_{\Gamma_{b}} \chi \widehat{k} \widehat{\psi} d y=0$.

Then, from (49) and (56), we have

$$
\begin{gathered}
\mu \int_{\mathbb{Q}} \frac{\partial u^{*}}{\partial z}\left(\frac{\partial \widehat{\psi}}{\partial z}-\frac{\partial u^{*}}{\partial z}\right) d y d z+\mu \alpha \wedge^{2} \int_{\mathbb{Q}} u^{*}\left(\widehat{\psi}-u^{*}\right) d y d z \\
-\int_{\mathbb{Q}} \widehat{f}\left(\widehat{\psi}-u^{*}\right) d y d z+\int_{\Gamma_{b}} \widehat{k}\left(|\widehat{\psi}|-\left|u^{*}\right|\right) d y \geq 0,
\end{gathered}
$$

which gives (47).

Theorem 9. With the same assumptions as Theorems 5 and 6, the pair $\left(u^{*}, p^{*}\right)$ satisfies

$$
\begin{gathered}
p^{*} \in H^{1}\left(\Gamma_{b}\right), \\
-\mu \frac{\partial^{2} u_{i}^{*}}{\partial z^{2}}+\frac{\partial p^{*}}{\partial y_{i}}+\mu \alpha \wedge^{2} u_{i}^{*}=\widehat{f}_{i} i n L^{2}\left(\Gamma_{b}\right) .
\end{gathered}
$$

Proof. We choose in (24) $\widehat{\varphi}_{3}=\widehat{u}_{3}^{\varepsilon} \pm \psi$ with $\psi$ in $H_{0}^{1}(\mathbb{Q})$ and $\widehat{\varphi}_{i}=\widehat{u}_{i}^{\varepsilon}$ (for $i=1,2$ ); we give (34). Using the convergence limit ((38) and (40)-(42)) in (34), we find

$$
\int_{\mathbb{Q}} p^{*} \frac{\partial \psi}{\partial z} d y d z=0, \quad \forall \psi \in H_{0}^{1}(\mathbb{Q})
$$

then,

$$
\frac{\partial p^{*}}{\partial z}=0 \text { in } H^{-1}(\mathbb{Q})
$$

Choosing $\widehat{\varphi}_{i}=\widehat{u}_{i}^{\varepsilon} \pm \psi($ for $i=1,2)$ with $\psi_{i}$ in $H_{0}^{1}(\mathbb{Q})$ and $\widehat{\varphi}_{3}=\widehat{u}_{3}^{\varepsilon}$ leads to

$$
\begin{aligned}
& \sum_{i, j=1}^{2} \int_{\mathbb{Q}}\left(\varepsilon^{2} \mu\left(\frac{\partial \widehat{u}_{i}^{\varepsilon}}{\partial y_{j}}+\frac{\partial \widehat{u}_{j}^{\varepsilon}}{\partial y_{i}}\right)-p \wedge^{\varepsilon} \delta_{i j}\right) \frac{\partial \psi}{\partial y_{j}} d y d z \\
& \quad+\sum_{i=1}^{2} \int_{\mathbb{Q}} \mu\left(\frac{\partial \widehat{u}_{i}^{\varepsilon}}{\partial z}+\varepsilon^{2} \frac{\partial \widehat{u}_{3}^{\varepsilon}}{\partial y_{i}}\right) \frac{\partial \psi}{\partial z} d y d z+\sum_{i=1}^{2} \int_{\mathbb{Q}} \mu \alpha \wedge^{2} \widehat{u}_{i}^{\varepsilon} \psi_{i} d y d z \\
& =\sum_{i=1}^{2} \int_{\mathbb{Q}} \widehat{f}_{i} \psi d y d z .
\end{aligned}
$$

Using (38), (39), (41), and (43) and choosing $\psi_{1}=0$ and $\psi_{2} \in H_{0}^{1}(\mathbb{Q})$ and then with $\psi_{2}=0$ and $\psi_{1}$ in $H_{0}^{1}(\mathbb{Q})$, we find

$$
\begin{aligned}
& \sum_{i=1}^{2} \int_{\mathbb{Q}}-p^{*} \frac{\partial \psi_{i}}{\partial y_{i}} d y d z+\sum_{i=1}^{2} \int_{\mathbb{Q}} \mu \frac{\partial u_{i}^{*}}{\partial z} \frac{\partial \psi_{i}}{\partial z} d y d z+\sum_{i=1}^{2} \int_{\mathbb{Q}} \mu \alpha \wedge^{2} u_{i}^{*} \psi_{i} d y d z \\
& \quad=\sum_{i=1}^{2} \int_{\mathbb{Q}} \widehat{f}_{i} \psi_{i} d y d z .
\end{aligned}
$$

By Green's formula, we deduce

$$
\begin{aligned}
\int_{\mathbb{Q}} & -\mu \frac{\partial^{2} u_{i}^{*}}{\partial z^{2}} \psi_{i} d y d z+\int_{\mathbb{Q}} \frac{\partial p^{*}}{\partial y_{i}} \psi_{i} d y d z+\mu \alpha \wedge^{2} \int_{\mathbb{Q}} u_{i}^{*} \psi_{i} d y d z \\
= & \int_{\mathbb{Q}} \widehat{f}_{i} \psi_{i} d y d z
\end{aligned}
$$

Then,

$$
-\mu \frac{\partial^{2} u_{i}^{*}}{\partial z^{2}}+\frac{\partial p^{*}}{\partial y_{i}}+\mu \alpha \wedge^{2} u_{i}^{*}=\widehat{f}_{i}, \text { in } H^{-1}(\mathbb{Q}) \quad(i=1,2) .
$$

To prove $p^{*} \in H^{1}\left(\Gamma_{b}\right)$, we see from Theorem 6 that $p^{*}$ does not depend on $z$. Then, as in [16], we choose $\psi_{i}(y, z)$ 
$=z(z-h(y)) \gamma(y)$ in (63); with $\gamma \in H_{0}^{1}\left(\Gamma_{b}\right)$, we obtain

$$
\begin{gathered}
\int_{\Gamma_{b}} \int_{0}^{h(y)}-p^{*} \frac{\partial}{\partial y_{i}}(z(z-h(y))) \gamma(y) d y d z \\
\quad+\int_{\Gamma_{b}} \int_{0}^{h(y)} \mu \frac{\partial u_{i}^{*}}{\partial z}(2 z-h(y)) \gamma(y) d y d z \\
\quad+\int_{\Gamma_{b}} \int_{0}^{h(y)} \mu \alpha \wedge^{2} u_{i}^{*}(z(z-h(y)) \gamma(y)) d y d z \\
=\int_{\Gamma_{b}} \int_{0}^{h(y)} \hat{f}(y, z)(z(z-h(y)) \gamma(y)) d y d z .
\end{gathered}
$$

Using the Green formula, we deduce

$$
\begin{gathered}
\frac{1}{6} \int_{\Gamma_{b}} p^{*} \frac{\partial\left(h^{3} \gamma(y)\right)}{\partial y_{i}} d y-2 \mu \int_{\Gamma_{b}} \int_{0}^{h(y)} u_{i}^{*}(y, z) \gamma(y) d y d z \\
\quad+\int_{\Gamma_{b}} \int_{0}^{h(y)} \mu \alpha \wedge^{2} u_{i}^{*}(z(z-h(y)) \gamma(y)) d y d z \\
=\int_{\Gamma_{b}} \int_{0}^{h(y)} \hat{f}(y, z) z(z-h(y)) \gamma(y) d y d z .
\end{gathered}
$$

Then,

$$
\begin{aligned}
& \frac{1}{6} \int_{\Gamma_{b}} p^{*} \frac{\partial\left(h^{3} \gamma\right)}{\partial y_{i}} d y-2 \mu \int_{\Gamma_{b}} h(y) \tilde{u}_{i}^{*} \gamma(y) d y+\int_{\Gamma_{b}} \mu \alpha \wedge^{2} \widehat{u}_{i}^{*} \gamma(y) d y \\
& \quad=\int_{\Gamma_{b}} \tilde{f} \cdot \gamma(y) d y
\end{aligned}
$$

where

$$
\begin{aligned}
\tilde{u}_{i}^{*}(x) & =\frac{1}{h(y)} \int_{0}^{h(y)} u_{i}^{*}(y, z) d z, \widehat{u}_{i}^{*}=\int_{0}^{h(y)} u_{i}^{*}(y, z) z(z-h(y)) d z, \tilde{f}_{i} \\
& =\int_{0}^{h(y)} \widehat{f}_{i}(z(z-h(y))) d z,
\end{aligned}
$$

whence

$$
-\frac{h^{3}}{6} \frac{\partial p^{*}}{\partial y_{i}}-2 \mu h(y) \tilde{u}_{i}^{*}+\mu \alpha \wedge^{2} \widehat{u}_{i}^{*}=\tilde{f}_{i} \text { in } H^{-1}\left(\Gamma_{b}\right) .
$$

As $f_{i} \in L^{2}(\mathbb{Q})$ and $u_{i}^{*} \in V_{z}$ then in $L^{2}(\mathbb{Q})$, therefore $\tilde{f}_{i}, \tilde{u}_{i}^{*}$, and $\widehat{u}_{i}^{*}$ are in $L^{2}\left(\Gamma_{b}\right)$. In addition, from (70), we get $p^{*}$ in $H^{1}\left(\Gamma_{b}\right)$. So as $f_{i}$ belongs to $L^{2}(\mathbb{Q})$ from $(61)$, we have $\partial^{2} u_{i}^{*} /$ $\partial z^{2} \in L^{2}(\mathbb{Q})$, whence (59) holds, and we also have $\partial u_{i}^{*} / \partial z$ in $V_{z}$.

Theorem 10. Suppose that the assumptions of the previous theorem hold; then, the solution $\left(u^{*}, p^{*}\right)$ satisfies the weak generalized equation of Reynolds:

$$
\begin{gathered}
\int_{\Gamma_{b}}\left[\frac{h^{3}}{12 \mu} \nabla p^{*}+h{\widetilde{u_{i}}}^{*}(y)-\frac{h}{2} s^{*}(y)+\widehat{\alpha}\left(\frac{h}{2} U^{*}(y, h)-\int_{0}^{h} U^{*}(y, t) d t\right)\right. \\
+\tilde{F}(y)] \nabla \varphi(y) d y=0, \quad \forall \varphi \in H^{1}(\mathbb{Q}) .
\end{gathered}
$$

where

$$
\begin{aligned}
& \tilde{F}(y)=\frac{1}{\mu} \int_{0}^{h(y)} F(y, t) d t-\frac{h}{2 \mu} F(y, h), U^{*}(y, t)=\int_{0}^{t} \int_{0}^{\zeta} u_{i}^{*}(y, \theta) d \theta d \zeta, \\
& F(y, t)=\int_{0}^{t} \int_{0}^{\zeta} \widehat{f}_{i}(y, \theta) d \theta d \zeta, s^{*}(y)=u^{*}(y, 0) \text { and } \tau^{*}(y)=\frac{\partial u^{*}}{\partial z}(y, 0) .
\end{aligned}
$$

Proof. By integrating expression (59) from 0 to $z$, it becomes

$$
\begin{gathered}
-\mu u_{i}^{*}(y, z)+\mu u_{i}^{*}(y, 0)+\mu z \frac{\partial u_{i}^{*}}{\partial z}(y, 0)+\frac{z^{2}}{2} \frac{\partial p^{*}}{\partial y_{i}} \\
+\mu \alpha \wedge^{2} \int_{0}^{z} \int_{0}^{\zeta} u_{i}^{*}(y, t) d t d \zeta=\int_{0}^{z} \int_{0}^{t} \widehat{f}_{i} d t d \zeta .
\end{gathered}
$$

In particular for $z=h$, we obtain

$\mu s^{*}(y)+\mu h \tau^{*}(y)+\frac{h^{2}}{2} \frac{\partial p^{*}}{\partial y_{i}}+\mu \alpha \wedge^{2} \int_{0}^{h} \int_{0}^{\zeta} u_{i}^{*}(y, t) d t d \zeta=\int_{0}^{h} \int_{0}^{\zeta} \hat{f}_{i}(y, t) d t d \zeta$.

Integrating (73) from 0 to $h$, we get

$$
\begin{aligned}
& -\mu \int_{0}^{h} u_{i}^{*}(y, t) d t+\mu h s^{*}(y)+\frac{h^{2} \mu}{2} \tau^{*}(y)+\frac{h^{3}}{6} \frac{\partial p^{*}}{\partial y_{i}} \\
& +\mu \alpha \wedge^{2} \int_{0}^{h} \int_{0}^{\zeta} \int_{0}^{t} u_{i}^{*}(y, \theta) d \theta d t d \zeta=\int_{0}^{h} \int_{0}^{\zeta} \int_{0}^{t} \widehat{f}_{i}(y, \theta) d \theta d t d \zeta .
\end{aligned}
$$

As $\tilde{u}_{i}^{*}(y)=1 / h \int_{0}^{h(y)} u_{i}^{*}(y, t) d t$, we have

$$
\begin{aligned}
& -\mu h \tilde{u}_{i}^{*}(y)+\mu h s^{*}(y)+\mu \frac{h^{2}}{2} \tau^{*}(y)+\frac{h^{3}}{6} \frac{\partial p^{*}}{\partial y_{i}} \\
& +\mu \alpha \wedge^{2} \int_{0}^{h} \int_{0}^{\zeta} \int_{0}^{t} u_{i}^{*}(y, \theta) d \theta d t d \zeta=\int_{0}^{h(y)} F(y, t) d t .
\end{aligned}
$$

From (74)-(76), we deduce (71), which ends the proof requested.

Theorem 11. Suppose that the assumptions of the previous theorem hold; then, the solution $\left(u^{*}, p^{*}\right)$ of the limit problem (45) is unique in $V_{z} \times L_{0}^{2}(\mathbb{Q})$.

Proof. Suppose that problem (47) admits two solutions that we denote by $\left(u^{*, 1}, p^{*, 1}\right)$ and $\left(u^{*, 1}, p^{*, 2}\right)$. Using classical techniques, we choose in (47) $\widehat{\varphi}=u^{*, 1}$ and then $\widehat{\varphi}=u^{*, 2}$ as test 
functions; then, by summing the two inequalities obtained, we find

$$
\sum_{i=1}^{2} \mu \int_{\mathbb{Q}}\left|\frac{\partial}{\partial z}\left(u_{i}^{*, 1}-u_{i}^{*, 2}\right)\right|^{2} d y d z+\sum_{i=1}^{2} \mu \alpha \wedge^{2} \int_{\mathbb{Q}}\left|u_{i}^{*, 1}-u_{i}^{*, 2}\right|^{2} d y d z \leq 0 .
$$

Then,

$$
\left\|\frac{\partial}{\partial z}\left(u^{*, 1}-u^{*, 2}\right)\right\|_{L^{2}(\mathbb{Q})}^{2}+\left\|u^{*, 1}-u^{*, 2}\right\|_{L^{2}(\mathbb{Q})}^{2} \leq 0 .
$$

Consequently, this last inequality ensures the uniqueness of the solution $u^{*}$ in $V_{z}$.

Similarly, we take in the Reynolds equation (71) the pressure value $p^{*}=p^{*, 1}$ and then $p^{*}=p^{*, 2}$, respectively, at the end by subtracting the equations obtained; it becomes

$$
\int_{\Gamma_{b}} \frac{h^{3}}{12 \mu} \nabla\left(p^{*, 1}-p^{*, 2}\right) \nabla \varphi d y=0 .
$$

Finally, by performing the change of variable $\varphi=p^{*, 1}-$ $p^{*, 2}$ and then by the Poincaré inequality, we obtain

$$
\left\|p^{*, 1}-p^{*, 2}\right\|_{H^{1}\left(\Gamma_{b}\right)}=0 .
$$

We deduce the desired result.

\section{Conclusions}

The purpose of this paper is to study the asymptotic convergence of an incompressible Brinkman-type fluid in thin medium with the Tresca friction on the bottom surface. One of the objectives of this study is to obtain a twodimensional equation that allows a reasonable description of the phenomenon occurring in the three-dimensional domain by passing the limit to 0 on the small thickness of the domain (3D). We show the existence and uniqueness results of the weak solution. We proceed to the study of the convergence analysis. To do this, we use the scale change following the third component and new unknowns to conduct the study on a fixed domain. Then, we prove different inequalities for the solution $\left(u^{\varepsilon}, p^{\varepsilon}\right)$. Finally, these estimates allow us to have the limit problem and establish the uniqueness of the solution.

\section{Data Availability}

No data were used to support this study.

\section{Conflicts of Interest}

The authors declare that they have no conflicts of interest.

\section{References}

[1] M. Billen, "Darcys law-flow in a porous medium," 2021, https://geo.libretexts.org/@go/page/3528.
[2] H. Brinkman, "A calculation of the viscous force exerted by a flowing fluid on a dense swarm of particles," Applied Scientific Research, vol. A1, pp. 27-34, 1949.

[3] J. L. Auriault, "On the domain of validity of Brinkmans equation," Transport in Porous Media, vol. 79, no. 2, pp. 215-223, 2009.

[4] L. Durlofsky and J. F. Brady, "Analysis of the Brinkman equation as a model for flow in porous media," Physics of Fluids, vol. 30, no. 11, p. 3329, 1987.

[5] N. Ho, K. Leiderman, and S. Olson, "A three-dimensional model of flagellar swimming in a Brinkman fluid," Journal of Fluid Mechanics, vol. 864, pp. 1088-1124, 2019.

[6] Z. A. Khana, S. U. Haq, T. S. Khan, I. Khan, and K. S. Nisar, "Fractional Brinkman type fluid in channel under the effect of MHD with Caputo-Fabrizio fractional derivative," Alexandria Engineering Journal, vol. 59, no. 5, pp. 2901-2910, 2020.

[7] M. Liu, Biodegradation of chlorobenzenes underaerobic and anaerobic conditions in model systems, [M.S. Thesis], A Thesis Submitted to the Johns Hopkins University in Conformity with the Optional Requirement of the Degree of Master of Science, Baltimore, Maryland, 2017.

[8] M. Dilmi, H. Benseridi, and A. Saadallah, "Asymptotic analysis of a Bingham fluid in a thin domain with Fourier and Tresca boundary conditions," Advances in Applied Mathematics and Mechanics, vol. 6, no. 6, pp. 797-810, 2014.

[9] A. Saadallah, H. Benseridi, and M. Dilmi, “Asymptotic convergence of a generalized non-Newtonian fluid with Tresca boundary conditions," Acta Mathematica Scientia, vol. 40B, no. 3, pp. 700-712, 2020.

[10] L. Consiglieri, "Stationary solutions for a Bingham flow with nonlocal friction," Pitman Research Notes in Mathematics, vol. 274, pp. 237-243, 1992.

[11] L. Consiglieri and J. F. Rodrigues, "Steady-state Bingham flow with temperature dependent nonlocal parameters and friction," Free Boundary Problems, vol. 154, pp. 149-157, 2007.

[12] G. Bayada and K. Lhalouani, "Asymptotic and numerical analysis for unilateral contact problem with Coulombs friction between an elastic body and a thin elastic soft layer," Asymptotic Analysis, vol. 25, pp. 329-362, 2001.

[13] G. Duvaut and J.-L. Lions, Les Inéquations en Mécanique et en Physique, Travaux et Recherches Mathématiques, Dunod, Paris, 1972.

[14] E. D. Tabacman and D. A. Tarzia, "Sufficient and/or necessary condition for the heat transfer coefficient on $\Gamma_{1}$ and the heat flux on $\Gamma_{2}$ to obtain a steady-state two-phase Stefan problem," Journal of Differential Equations, vol. 77, pp. 16-37, 1989.

[15] R. Temam and I. Ekeland, Analyse Convexe et Problème Variationnels, Gauthier-Villars, Dunod, Paris, 1974.

[16] M. Boukrouche and R. Elmir, "Asymptotic analysis of a nonNewtonian fluid in a thin domain with Tresca law," Nonlinear Analysis: Theory Methods \& Applications, vol. 59, no. 1-2, pp. 85-105, 2004. 